\title{
Cloud-cloud collision in the Sgr B2 molecular cloud complex
}

\author{
Masato Tsuboi ${ }^{1}$, Atsushi Miyazaki ${ }^{2}$, and Toshihiro Handa ${ }^{3}$ \\ ${ }^{1}$ Institute of Space and Astronautical Science, Japan Aerospace Exploration Agency, \\ 3-1-1 Yoshinodai, Chuo-ku, Sagamihara, Kanagawa 252-5210, Japan \\ email: tsuboi@vsop.isas.jaxa.jp \\ ${ }^{2}$ Korean VLBI Network, Korea Astronomy and Space Science Institute, 776 Daedeokdae-ro, \\ Yuseon-gu, Daejeon 305-348, Republic of Korea \\ ${ }^{3}$ Graduate School of Science and Engineering, Kagoshima University, Kagoshima, Kagoshima, \\ 890-0065, Japan
}

\begin{abstract}
We present results from a high-resolution wide-field imaging observation of the central molecular zone $(\mathrm{CMZ})$ in $\mathrm{H}^{13} \mathrm{CO}^{+} J=(1-0)$ and $\mathrm{SiO} v=0, J=(2-1)$ emission lines by using the Nobeyama $45-\mathrm{m}$ telescope in order to depict the high-density molecular gas mass distribution and explore molecular gas affected by interstellar shocks. We found a candidate for ongoing cloud-cloud collision in the Sgr B2 complex. This is identified as a hollow paraboloid-like structure in the $l-b-v$ data cube of both emission lines. The central part of the feature is denser and warmer than the outer envelope and contains a vast amount of shocked molecular gas. These properties are consistent with those expected from simulations of cloud-cloud collisions in the CMZ.
\end{abstract}

Keywords. central molecular zone, Sgr B2 molecular cloud, young massive clusters

\section{Introduction}

In the recent two decades, the young massive clusters (YMCs) the Arches cluster, the Quintuplet cluster, and the Central cluster have been found by IR observations in the central molecular zone (CMZ) (e.g., Figer et al. 1999). Although it is an open question as to what mechanism is responsible for the formation of such clusters in the region, the dense, warm, and turbulent molecular clouds in the CMZ are presumably cradles of these clusters. Because the CMZ is very crowding, the YMCs must be forming in conditions that are affected by external influences, such as interactions with SNRs and cloud-cloud collisions (e.g., Morris 1993, Hasegawa et al. 1994).

\section{Observation and results}

We performed simultaneous observations of the CMZ including the Sgr A and Sgr B2 complexes in $\mathrm{H}^{13} \mathrm{CO}^{+} J=(1-0)$ and $\mathrm{SiO} v=0, J=(2-1)$ emission lines using the Nobeyama 45-m telescope (also see Tsuboi et al. 2011). Because SiO emission line is a tracer of shocked molecular gas and $\mathrm{H}^{13} \mathrm{CO}^{+}$emission line is a tracer of the mass of molecular gas with $n\left(\mathrm{H}_{2}\right)>10^{5} \mathrm{~cm}^{-3}, T(\mathrm{SiO}) / T\left(\mathrm{H}^{13} \mathrm{CO}^{+}\right)$indicates the existence of shock in the molecular clouds. We found a significant difference between the Sgr A and Sgr B2 molecular cloud complexes in the ratio. The Sgr B2 complex has several expanding shell-like structures. The expanding shells have very wide velocity width up to $\sim 100 \mathrm{~km} \mathrm{~s}^{-1}$. Although the ratios in the Sgr B2 molecular cloud itself are as low as $1-3$, the shells have significantly high ratio $(4-8)$, which shows that the shells contain a vast amount of shocked molecular gas. They are presumably originated with successive 

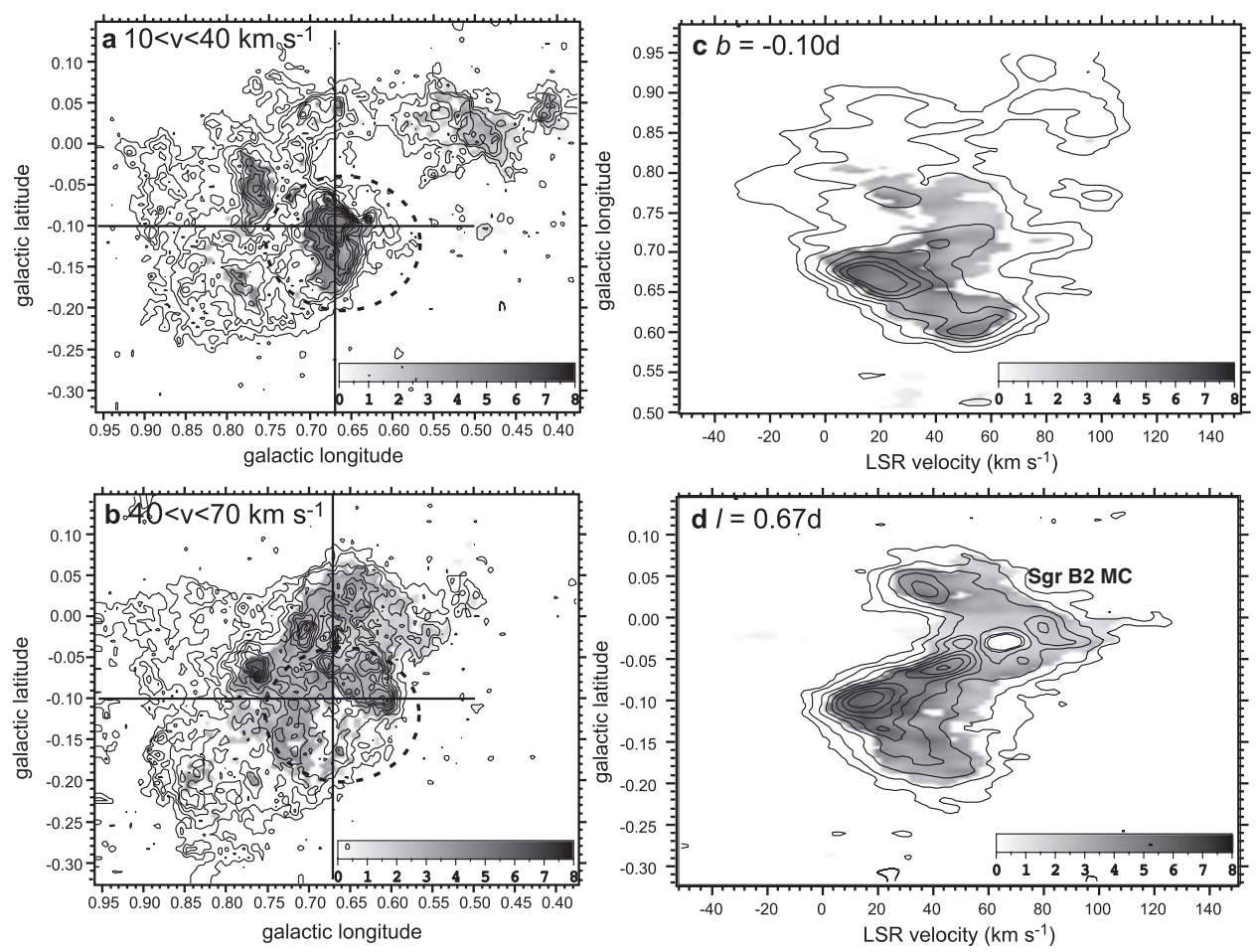

Figure 1. a and b Velocity integrated maps in $\mathrm{SiO}$ emission line (contours) and the $T(\mathrm{SiO}) / T\left(\mathrm{H}^{13} \mathrm{CO}^{+}\right)$(gray scale). c The Galactic longitude-LSR velocity diagram. d The Galactic latitude-LSR velocity $(b-v)$ diagram.

supernovae in the CMZ. On the other hand, there is no such large shells with a high brightness temperature ratio in the Sgr A complex.

We also found a peculiar feature which is identified as a filled center and circular feature south of the Sgr B2 HII region in the velocity integrated maps of the SiO line shown in Figure $1 \mathrm{a}$ and $1 \mathrm{~b}$. Figure 1c and 1d show the Galactic longitude-LSR velocity diagram of the brightness temperature ratio along $b=-0.10^{\circ}$ and the Galactic latitude-LSR velocity $(b-v)$ diagram along $l=0.67^{\circ}$ of the ratio, respectively. They show that this feature is a hollow paraboloid-like structure in the $l-b-v$ data cube of both emission lines. The brightness temperature ratio on the central part of the feature is up to $\sim 8$, which is much higher than those on the outer envelope, which are $2-3$. This shows that vast shocked molecular gas exists in the central part. The central part has wider velocity component rather than the outer part. It is hard to make the ratio distribution and kinetic structure by a single explosive event at the center position or even by a succession of such events. They indicate the origin is different from those of the expanding shells mentioned above. One possible mechanism explaining the peculiar feature may be cloud-cloud collisions (e.g., Habe \& Ohta 1992) in the following way. When the molecular cloud collided with a smaller cloud, the central part of the collision created a shock wave and was decelerated. In contrast, the outer part was not strongly affected by the collision. Then the velocity of the central part should be shifted relative to the outer part because of the deceleration due to the merging. The velocity width should be larger than that of the outer part because of the shock wave. These expected characteristics are indeed seen in Figure 1. 


\section{References}

Figer, D. F., McLean, I. S., \& Morris, M. 1999, APJ 514, 202

Habe, A. \& Ohta, K. 1992, PASJ 44, 203

Hasegawa, T., Sato, F., Whiteoak, J. B., \& Miyawaki, R. 1994, APJ 429, L77

Morris, M. 1993, APJ 408, 496

Tsuboi, M., Tadaki, K-I., Miyazaki, A., \& Handa, T. 2011, PASJ 63, 763 